\title{
Interferometry Evaluation of Precorneal Tear Film Lipid Layer After Intense Pulsed Light in Evaporative Dry Eye Disease owing to Meibomian Gland Dysfunction: A Randomized, Single Masked, Sham-Controlled Study.
}

Yilin Song MD 1,2, Sile Yu, MD MPH CPH 3 , Xingru He MPH MBA DrPH ${ }^{3}$, Lanting Yang MD 1,2, Yi Wu MD 1,2, Guanghao Qin MD 1,2, Qing Zhang MD 1,2, Gagan Deep Singh Talwar MD 4, Ling Xu MD ${ }^{1}$, Jonathan E Moore MD, PhD,5,6, Wei He MD, PhD*1,3, Emmanuel Eric Pazo MD, MSc, $\mathrm{PhD}^{* 1,3}$

1 He Eye Specialist Hospital, No.128 North Huanghe Street, Shenyang, China.

2 The Second Affiliated Hospital of Dalian Medical University, Dalian, China.

3 He University, Shenyang, China.

4 Tianjin Medical University, Tianjin, China.

5 Cathedral Eye Clinic, 89-91 Academy Street, Belfast, United Kingdom.

6. Biomedical Sciences Research Institute, University of Ulster, Coleraine, United Kingdom

* Correspondence: Wei He \& Emmanuel Eric Pazo

\begin{abstract}
:
Background: Inadequate meibomian glands (MGs) secretion can lead to dry eye signs and symptoms. Tear film lipid layer (TFLL) secreted by MGs protects and prevents rapid evaporation of tear film. Our purpose was to assess TFLL alteration and function in patients with evaporative dry eye (EDE) using tear interferometry after optimal pulse light technology (OPT) intense pulsed light (IPL). Methods: This prospective randomized examiner-masked sham- controlled study included 86 participants (142 eyes) with DED. IPL or sham procedure was performed on day 0, 21, and 42. Ocular Surface Disease Index (OSDI), non-invasive breakup time (NITBUT), interferometric fringe pattern determined TFLL quality, fluorescein staining (FS), and meibum gland (MG) were assessed at day 0, 21, 42 and 3-month. Results: At 3-month, TFLL, NITBUT, MG drop-out, MG quality, MG expressibility, FS and OSDI improved significantly $(\mathrm{P}<0.05)$ in the IPL group, while the sham group had no significant improvements.
\end{abstract}


All DE parameters significantly correlated with the improvement in TFLL following IPL treatment. Additionally, artificial tears usage was significantly less in the IPL group from D-42 onwards. Conclusion: IPL treatment demonstrated the ability to improve TFLL quality and clinically reduced sign and symptoms of DED thereby reducing the frequency of artificial tears usage.

Keywords: dry eye disease, meibomian gland, tear stability; tear film lipid layer; interferometry; OSDI; intense pulse light; IPL

\section{Introduction:}

The prevalence of dry eye disease (DED) is common and can lead to ocular discomfort, reduced visual acuity, lowered quality of vision and life[1,2]. Epidemiological reports suggest that the prevalence of DED ranges from $5 \%$ to $50 \%$ in general population across the world [1,39]. The large variation in prevalence is due to the differences of diagnostic criteria, characteristics of investigated participants and etiological factors [10-12]. The most prevalent form of dry eye (DE) is evaporative dry eye (EDE), accounting up to two-thirds of all DE cases, mostly due to meibomian gland dysfunction (MGD) [13-15]. MGD is defined as "a chronic, diffuse abnormality of the meibomian gland, commonly characterized by terminal duct obstruction and/or qualitative/quantitative changes in the glandular secretion" by the International Workshop on MGD [16-18]. Meibomian glands are modified sebaceous glands that are situated within the upper and lower eyelid and their ducts terminate along the margins of the eyelids and secrete meibum; which directly contributes to the lipid component of tears [19]. The tear-film lipid layer (TFLL) is a thin outermost layer that envelops the tear film. This phase stabilizes the film as it decrease the surface tension and improves viscoelastic properties. Clinically, negative alterations to the TFLL can lead to symptomatic and clinical presentation of ocular surface disease, and inflammation accompanied by changes in the quality and quantity of TFLL[20]. The treatment of EDE and MGD consists of improving symptoms by enhancing the quality and quantity of meibum excretion[21-24]. Studies of lipid-containing eye drops, castor oil emulsions have shown promising results in mimicking and restoring the TFLL of tears [25,26]. Preservative-free drops, omega-3 fatty acid supplementation, topical cyclosporine, serum tears, topical azithromycin, oral doxycycline, cholinergics, lacrimal plug, lid massage and expression, warm compresses, amniotic membrane biologic corneal bandage lens, intense pulse light (IPL) have been demonstrated to improve the signs and symptoms of 
DE[27]. However, treatment discontinuation often leads to relapse of signs and symptoms DED as the positive effects of these treatments are not sustained for long period of time[28,29].

Intense pulsed light (IPL) treatment is commonly used for dermatological conditions and lesions[11]. IPL treatment utilizes a noncoherent polychromatic light source with wavelength spectrum of $500-1200 \mathrm{~nm}$ on the cutaneous facial sebaceous glands. This photothermal effect is thought to decrease inflammation and stimulate the meibomian glands. IPL treatment with or without MG expression has been proven to be an effective therapy for the improvement of signs and symptoms of DE due to MGD [11]. Although improvements after IPL treatment on the signs and symptoms of DE have been documented, the impact of IPL treatment upon precorneal tear film lipid layer has not been documented before. The purpose of this study is to assess the improvement in the TFLL and its subsequent impact upon TFLL interferometry patterns in patients with evaporative DE due to MGD.

\section{Materials and Methods}

\subsection{Study design and participants}

This study was conducted in compliance with the Institutional Review Board of He Eye Specialist Hospital, Shenyang, China in accordance with the tenets of the Declaration of Helsinki (approval number: IRB2019.K002.01). Consecutive subjects were recruited from He Eye Specialist Hospital, Shenyang outpatient department. Informed written consent was obtained from all participants after careful explanation of the nature and possible consequences of the study. Data from these participants was collected at the anterior segment department, a specialized ocular surface unit between January 2019 to January 2020.

Inclusion criteria comprised the following: (i) age $\geq 18$ years, (ii) Fitzpatrick skin types 1 to 4, (iii) able and willing to comply with the treatment/follow-up schedule and requirements, (iv) diagnosis of DE based on (a) ocular symptoms, (b) non-invasive tear film breakup time (NITBUT) of $\leq 5 \mathrm{sec}$, and fluorescein staining score of $\geq 1$ (on a scale of 0 to 9 ) according to the van Bijsterveld method [30]; (iv) visualisation of meibomian glands on each lower and upper meibography image, and (v) bilateral diagnosed at any stages of MGD, according to the International Workshop on MGD [31].

Exclusion criteria: Fitzpatrick skin type 5, any eyelid structural abnormality, any intraocular inflammation, ocular surgery, or ocular trauma in the past 6 months, ocular infection or allergy, pigmented lesion in the treatment zone, any systematic diseases or medication that may lead to dry eye disease, pterygium, corneal neovascularization, glaucoma, rheumatic immune systemic diseases, history of herpes zoster infection, skin cancer, pregnancy or breastfeeding, fluorescein allergy and contact lens wears were excluded from the study.

Initially, 106 (212 eyes) participants were eligible and randomized (1:1) into IPL treatment group and sham treatment group. Finally, 86 participants (172 eyes) were included in the final 
analysis, which exceeded the sample size requirements for the preferred study power. Power calculations were conducted with NITBUT as the designated outcome, and showed that a minimum of 30 participants was required in the IPL treatment group and a minimum of 30 participants was required in the sham treatment group, to detect a clinically significant difference of 3-4 s in pair- wise comparisons, at $90 \%$ power $(\beta=0.2)$ and a two-sided statistical significance level of $5 \%(\alpha=0.05)$, with the SD of normal values being estimated to be approximately $5-7 \mathrm{~s}$.

\subsection{Clinical evaluation}

TFLL interferometry. DR-1 (Kowa, Nagoya, Japan) was performed non-invasive TFLL quality with the Yokoi DE severity grading system; grade 1: somewhat grey colour, uniform distribution; grade 2: somewhat grey colour, non- uniform distribution; grade 3: a few colours, nonuniform distribution; grade 4: many colours, nonuniform distribution; TFLL interferometry[11].

NITBUT: Keratograph 5M (Oculus, Germany) is a clinical instrument that uses an infrared light source of wavelength $880 \mathrm{~nm}$ to assess the ocular surface, tear film and meibomian glands (http://www. oculus.de/)[32]. During each assessment time point, non-invasive first tear film breakup time using the Keratograph 5M (Oculus, Germany) topographer was measured three times consecutively and the median value was recorded.

Meibography (Meibo-score): Keratograph 5M (Oculus, Germany) was used to capture the upper and lower eyelids were turned over and the meibomian glands were observed. Partial or complete loss of the meibomian glands was scored using the following grades (meibo-score) for each eyelid: grade 0 , no loss of meibomian glands; grade 1 , area loss was less than one third of the total meibomian gland area; grade 2, area loss was between one third and two thirds; grade 3, area loss was more than two thirds [33].

Meibomian gland function: The quality of meibum quality and meibomian gland expressibility of the upper eyelid were assessed. (i) Meibum quality: Eight meibomian gland in the middle parts of the eyelid were assessed using a scale of $0-3$ for each gland: 0 , clear; 1 , cloudy; 2, cloudy and granular; and 3, thick (like toothpaste). (ii) Meibum expressibility: Five meibomian glands in the middle part were evaluated on a scale of $0-3 ; 0$ : all glands expressible; 1: 3-4 glands expressible; 2: 1-2 glands expressible; and 3: no glands expressible. The average scores of these eight glands were calculated as the total score.

Fluorescein staining (FS): In brief, corneal and conjunctival epithelial damage was evaluated by the double vital staining method. Two microliters of a preservative-free combination of $1 \%$ lissamine green and $1 \%$ sodium fluorescein were instilled into the conjunctival sac. The eye was sectionalized into 3 equal sections representing temporal conjunctiva, cornea, and nasal conjunctiva. Maximum staining score for each area was 3 point 
and the minimum were 0 points. Scores from all 3 sections were then added and reported on a scale of 0 (normal) to 9 (severe) $[17,22,28]$.

OSDI: Validated Chinese web-version of OSDI (Allergan Inc, Irvine, CA) was used to assess DE symptom frequency and the impact of these symptoms on vision-related function [34]. It contains 12 items, and the score can range from 0 (no symptoms) to 100 (severe symptoms) points; 0 to 12 represents normal, 13 to 22 represents mild DED, 23 to 32 represents moderate DED, and 33 or more represents severe DED.

Patient survey: Online question (How many times did you use preservative-free artificial tears yesterday?) was administered using a smartphone messaging platform to ascertain the frequency of artificial tears used during the past day by the participants in this study (e.g., once a day, twice a day and so on).

\subsection{Treatment}

The Toyos protocol was used in all treatments [35]. All patients had a minimum of 2 treatments, each separated by 3 weeks. IPL was performed using M22 IPL system with optimal pulse technology (OPT) (Lumenis Ltd., Yokneam, Israel). Its xenon lamp that emits IPL at 515$1200 \mathrm{~nm}$ and a 560-nm filter and OPT makes IPL pulses more stable and highly repeatable, therefore M22 IPL treatment is considered more effective in targeting meibomian glands[36]. The sapphire-cooled $6 \mathrm{~mm}$ cylindrical light guide set at a fluence of $10 \mathrm{~J} / \mathrm{cm} 2$ (Table 1). Each patient underwent a series of three OPT-IPL treatment or sham treatment sessions at 3-week intervals at day-0 (D-0), day-21 (D-21) and day-42 (D-42). Participants underwent clinical assessment as described above, at baseline (D-0) and after treatment at day-21 (D-21), day-42 (D-42) and 3-month (3-M). All participants were asked to continue their artificial tears use and not start or continue any other topical or systemic agent for DED or MGD during the course of this study (Figure 1). Before and after each treatment of OPT-IPL, all participants had a full ophthalmic examination, including uncorrected measurement of logarithm of the minimum angle of resolution (Snellen) best-corrected visual acuity (BCVA) at $4 \mathrm{~m}$ using the Early Treatment of Diabetic Retinopathy Study Chart, intraocular pressure (IOP) and corneal endothelial cell count (ECC).

Participants were randomly assigned to one of two treatment groups and underwent IPL treatment with 12 homogeneously spaced pulsed light to both eyes or sham treatment to both eyes, at days 0,21 , and 42 by a non-masked trained clinician, who was not involved in data collection process of this study. Randomisation was managed by computer-generated random number allocation to sequentially enrolled participants. The investigator involved in gathering data at days 0, 21, 42 and 3-months from all participant (IPL treatment and sham treatment group) was not involved in treatment allocation. During each visit at days 0, 21, and 42, participants eyes were protected with opaque goggles and ultrasound gel was applied 
generously on the patient's targeted skin area. Each participant in the IPL treatment group received 12 pulses of light bilaterally (with slightly overlapping applications) from the preauricular area and across the cheek. While participants allocated to the sham treatment group followed the same pre-treatment protocol with opaque goggles and ultrasound gel application and then a non-active IPL device was placed on the periocular area and was moved 12 times to simulate treating different areas of the periocular area, while an active IPL device was fired 12 times in the same room to imitate the acoustics of an active IPL device.

All participants were requested not to wear facial makeup and contact lens on treatment day and not to wear CL 3 weeks before baseline measurements and for the entire course of the study. To prevent possible facial pigmentation secondary to IPL treatment, participants were informed to avoid direct sun exposure for 1 month after each IPL treatment.

Table 1. Device parameters

\begin{tabular}{ll}
\hline Parameters & \\
\hline Manufacturer & Lumenis \\
Model Identifier & M22 \\
Year Produced & 2018 \\
Number \& Type of Emitters (laser or LED) & IPL \\
Wavelength and bandwidth [nm] & $590 \mathrm{~nm}$ \\
Pulse mode [CW or Hz, duty cycle] & Long pulse \\
Beam spot size at target [cm2] & $15^{*} 35 \mathrm{~mm}, 8^{*} 15 \mathrm{~mm}$ \\
Exposure duration [sec] & $4-20 \mathrm{~ms}$ \\
Radiant exposure [J/cm2] & $10-14 \mathrm{~J} / \mathrm{cm}^{2}$ \\
Radiant energy [J] & $52.5-73.5 \mathrm{~J}$ \\
Number of points irradiated & $12 \mathrm{shots}$ \\
Area irradiated [cm2] (Total treatment area & $5.25 \mathrm{~cm}^{2}$ per shot x 12 shots \\
size) & OPT ${ }^{\mathrm{TM}}$; SapphireCool ${ }^{\mathrm{TM}}$ \\
Application technique & $2 \mathrm{sessions,} 2$ weeks interval \\
Number and frequency of treatment sessions & \\
Total radiant energy over entire treatment & $1260-1764 \mathrm{~J}$ per session (calculated \\
course [J] & with 12 shots per session) \\
\hline
\end{tabular}




\section{Fig 1}

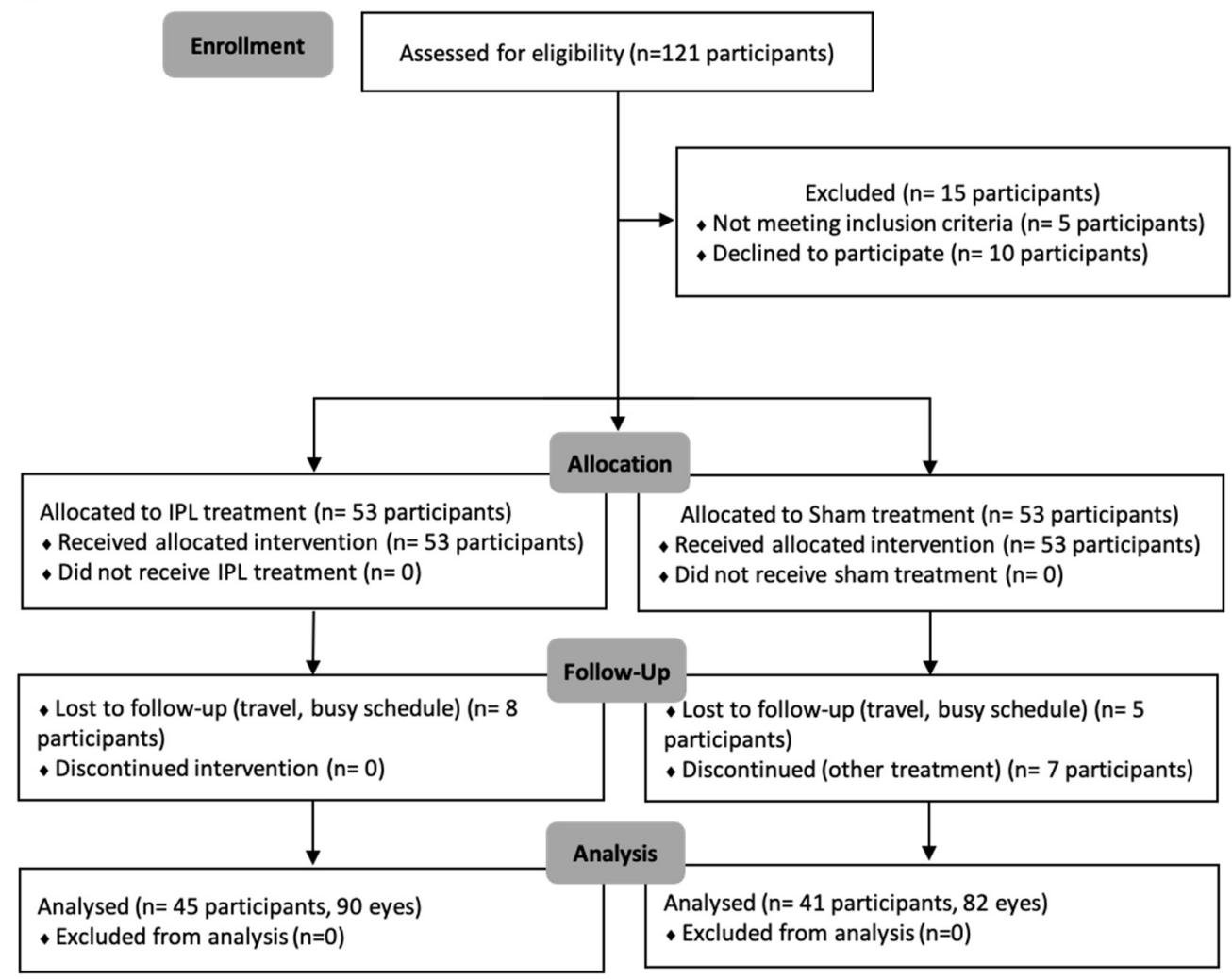

Figure 1. The flowchart of the experimental framework of this study, enrolment, randomization, intervention, follow-up and analysis.

\subsection{Statistical analysis}

Data are presented as mean \pm standard deviation and all analysis was performed using SPSS version 24 (SPSS Inc., Chicago, IL, USA. Data were found to be non-normally distributed with the Shapiro-Wilk test $(P<0.05)$, and nonparametric testing was therefore applied. A linear mixed model with Bonferroni post-hoc analysis was used to evaluate repeated measurements of continuous variables, including NITBUT, OSDI score, BCVA, ECC and IOP. Generalized linear mixed model analysis with Bonferroni post hoc analysis was used for repeated measurements of discrete variables, including the TFLL, FS score, MG assessments and ATD usage. For correlation analyses, Pearson's and Spearman's correlation analyses were used for continuous and discrete values, respectively. The level of statistical significance was set at $P<$ 0.05 .

\section{Results}

\subsection{Patient Characteristics}


The mean \pm SD age of IPL group of 45 participants (26 females, 19 males) was $28.16 \pm 3.59$ years (range, 21-34 years) and sham group of 41 participants (23 females, 18 males) was 28.07 \pm 3.71 years (range, 21-34 years). Participants in both groups had symptoms and signs of DED due to MGD. The demographics characteristics of participants in the study are presented in table 2. The final analysis consisted of 71 Asian adult participants (142 eyes) (Figure 1). Baseline clinical DED parameters did not differ between the IPL treatment and sham group (all $P>0.05$; Table 3).

Table 2. Demographic characteristics of participants in the study.

\begin{tabular}{llll}
\hline Demographic factors & IPL & Sham & $\boldsymbol{P}$ \\
\hline No. of participants (eyes) & $45(90)$ & $41(82)$ & - \\
Age, mean \pm SD (years) & $28.16 \pm 3.59$ & $28.07 \pm 3.71$ & 0.883 \\
Min, max & 21,34 & 21,34 & - \\
Sex, females (\%) & $26(58 \%)$ & $23(56 \%)$ & 0.825 \\
Year/s since dry eye, mean \pm & & & \\
SD & $3.32 \pm 1.51$ & $3.05 \pm 1.45$ & 0.227 \\
Min, max & 1,7 & 1,6 & - \\
AT usage per day (times) & $3.41 \pm 0.82$ & $3.18 \pm 0.72$ & 0.055 \\
Min, max & 2,5 & 2,5 & - \\
\hline
\end{tabular}

$P$ values were determined with Mann-Whitney $U$ test or Fisher's exact test. SD, standard deviations. IPL: intense pulse light; AT: artificial tear; *: statistically significant at $P<0.05$. 


\section{Fig 1}

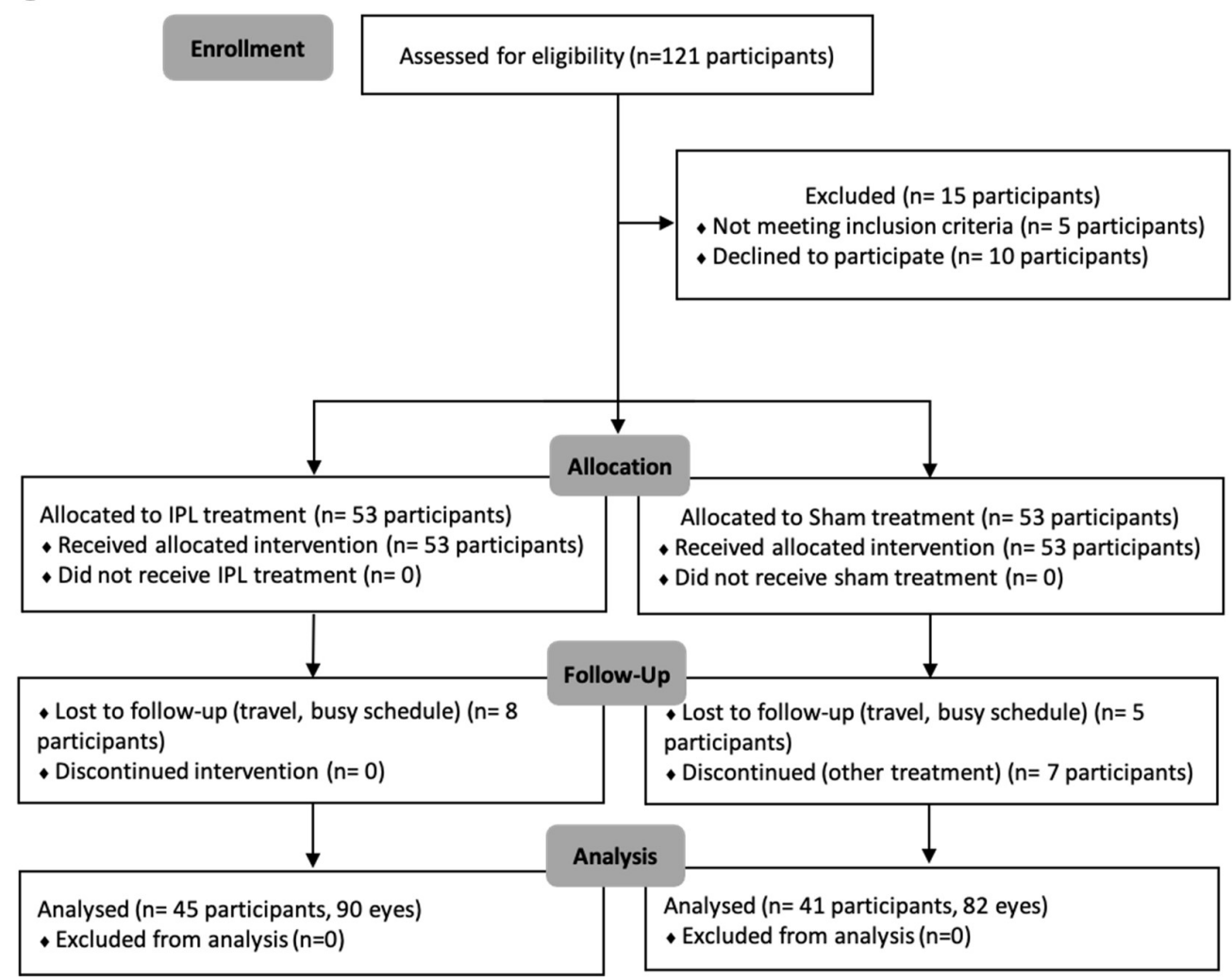

Figure. 1. Consolidated standards of reporting trials 2010 flow diagram.

Table 3. Characteristics of participants in intense pulsed light (IPL) treatment group and IPL sham (control) group at baseline and after treatment.

\begin{tabular}{|c|c|c|c|c|c|c|c|c|}
\hline Groups & $\begin{array}{l}\text { TFLL } \\
(1-5)\end{array}$ & $\begin{array}{l}\text { NITBU } \\
\text { T (sec) }\end{array}$ & $\begin{array}{l}\text { Meibo- } \\
\text { score (0- } \\
3)\end{array}$ & $\begin{array}{l}\text { MG } \\
\text { qualit } \\
\text { y (0-3) }\end{array}$ & $\begin{array}{l}\text { MG } \\
\text { expressio } \\
\text { n (0-3) }\end{array}$ & $\begin{array}{l}\text { FS (0- } \\
9)\end{array}$ & $\begin{array}{l}\text { OSDI } \\
(0-100)\end{array}$ & $\begin{array}{l}\text { AT } \\
\text { usage/24 } \\
\text { hrs }\end{array}$ \\
\hline \multicolumn{9}{|c|}{ D-O (Mean \pm SD) } \\
\hline IPL & $\begin{array}{l}3.27 \pm \\
0.67\end{array}$ & $\begin{array}{l}4.02 \\
0.76\end{array}$ & $1.73 \pm 0.44$ & $\begin{array}{l}2.54 \pm \\
0.50\end{array}$ & $2.14 \pm 0.63$ & $\begin{array}{l}3.30 \pm \\
1.19\end{array}$ & $\begin{array}{l}35.40 \pm \\
9.39\end{array}$ & $3.41 \pm 0.82$ \\
\hline Sham & $\begin{array}{l}3.18 \pm \\
0.61\end{array}$ & $\begin{array}{l}4.09 \\
0.72\end{array}$ & $1.68 \pm 0.47$ & $\begin{array}{l}2.51 \pm \\
0.50\end{array}$ & $2.11 \pm 0.59$ & $\begin{array}{l}3.23 \pm \\
1.06\end{array}$ & $\begin{array}{l}34.38 \pm \\
9.57\end{array}$ & $3.18 \pm 0.72$ \\
\hline$P$ value for & & & & & & & & \\
\hline $\begin{array}{l}\text { IPL } \\
\text { sham }\end{array}$ & 0.393 & 0.529 & 0.47 & 0.674 & 0.177 & 0.693 & 0.481 & 0.055 \\
\hline$F$ & 0.732 & 0.397 & 0.524 & 0.177 & 0.045 & 0.156 & 0.499 & 3.722 \\
\hline \multicolumn{9}{|c|}{ D-21 (Mean \pm SD) } \\
\hline IPL & $\begin{array}{l}2.94 \pm \\
0.71\end{array}$ & $\begin{array}{l}5.54 \\
1.08\end{array}$ & $1.24 \pm 0.64$ & $\begin{array}{l}2.26 \pm \\
0.68\end{array}$ & $1.77 \pm 0.64$ & $\begin{array}{l}3.26 \pm \\
1.14\end{array}$ & $\begin{array}{l}33.64 \pm \\
8.99\end{array}$ & $3.12 \pm 0.79$ \\
\hline
\end{tabular}






\begin{tabular}{|c|c|c|c|c|c|c|c|c|}
\hline IPL & $\begin{array}{l}1.84 \pm \\
0.62\end{array}$ & $\begin{array}{l}6.71 \\
0.99\end{array}$ & $1.12 \pm 0.61$ & $\begin{array}{l}1.26 \pm \\
0.74\end{array}$ & $1.20 \pm 0.74$ & $\begin{array}{l}2.98 \pm \\
1.04\end{array}$ & $\begin{array}{l}19.02 \pm \\
6.70\end{array}$ & $1.51 \pm 0.52$ \\
\hline Sham & $\begin{array}{l}3.28 \pm \\
0.63\end{array}$ & $\begin{array}{l}3.93 \\
0.73\end{array}$ & $1.74 \pm 0.44$ & $\begin{array}{l}2.61 \pm \\
0.49\end{array}$ & $2.10 \pm 0.53$ & $\begin{array}{l}3.34 \pm \\
0.96\end{array}$ & $\begin{array}{l}35.13 \pm \\
9.41\end{array}$ & $3.27 \pm 0.77$ \\
\hline $\begin{array}{l}P \text { value for } \\
\text { IPL vs. } \\
\text { sham }\end{array}$ & $\begin{array}{l}<0.001 \\
*\end{array}$ & $<0.001^{*}$ & $<0.001^{*}$ & $\begin{array}{l}<0.001 \\
*\end{array}$ & $<0.001^{*}$ & $0.018^{*}$ & $<0.001^{*}$ & $<0.001^{*}$ \\
\hline$F$ & $\begin{array}{l}226.63 \\
8\end{array}$ & 376.15 & 57.209 & $\begin{array}{l}194.89 \\
5\end{array}$ & 101.338 & 5.662 & 169.557 & 310.281 \\
\hline
\end{tabular}




\begin{tabular}{|c|c|c|c|c|c|c|c|c|}
\hline \multicolumn{9}{|l|}{$P$ value for } \\
\hline $\begin{array}{l}\text { IPL } \\
\text { baseline } \\
\text { vs. IPL 3- } \\
\text { month }\end{array}$ & $\begin{array}{l}<0.001 \\
*\end{array}$ & $<0.001^{*}$ & $<0.001^{*}$ & $\begin{array}{l}<0.001 \\
*\end{array}$ & $<0.001^{*}$ & 0.055 & $<0.001^{*}$ & $<0.001^{*}$ \\
\hline$F$ & $\begin{array}{l}220.66 \\
8\end{array}$ & 420.84 & 58.362 & $\begin{array}{l}186.24 \\
9\end{array}$ & 85.543 & 3.732 & 181.471 & 343.105 \\
\hline $\begin{array}{l}P \text { value for } \\
\text { sham }\end{array}$ & & & & & & & & \\
\hline $\begin{array}{l}\text { baseline } \\
\text { vs. sham 3- } \\
\text { month }\end{array}$ & 0.317 & 0.465 & 0.391 & 0.21 & 0.331 & 0.49 & 0.611 & 0.465 \\
\hline$F$ & 1.007 & 0.536 & 0.74 & 1.58 & 0.951 & 0.48 & 0.26 & 0.536 \\
\hline
\end{tabular}

* adjusted $P<0.05,{ }^{* *}$ adjusted $\mathrm{P}<0.001 ; \mathrm{D}-0$ : baseline; D-21: day-21; D-42: day-42; 3-M: 3-month; IPL: intense pulsed light; TFLL; tear film lipid layer; NITBUT: non-invasive breakup time; MG: meibum gland; FS: fluorescein staining; OSDI: ocular surface disease index; AT: artificial tears.

\subsection{Efficacy of IPL treatment}

Interferometric pattern (lipid layer grade) was used to grade TFLL quality from a scale of 1 to 5 . There was no significantly different $(P>0.05)$ in TFLL score at baseline between IPL $(3.17 \pm 0.67)$ and sham groups $(3.18 \pm 0.61)$. IPL group's TFLL significantly improved $(P<0.05)$ at day-21 (2.94 \pm 0.71 vs. $3.22 \pm 0.63)$, day-42 (1.92 \pm 0.69 vs. $3.27 \pm 0.65)$ and 3 -month $(1.84 \pm 0.62$ vs. $3.28 \pm 0.63$ ) in comparison to sham group (Figure 2). Additionally, along with TFLL scores, NITBUT was also significantly prolonged in the IPL group compared to the sham group at all assessment time points (Table 3).

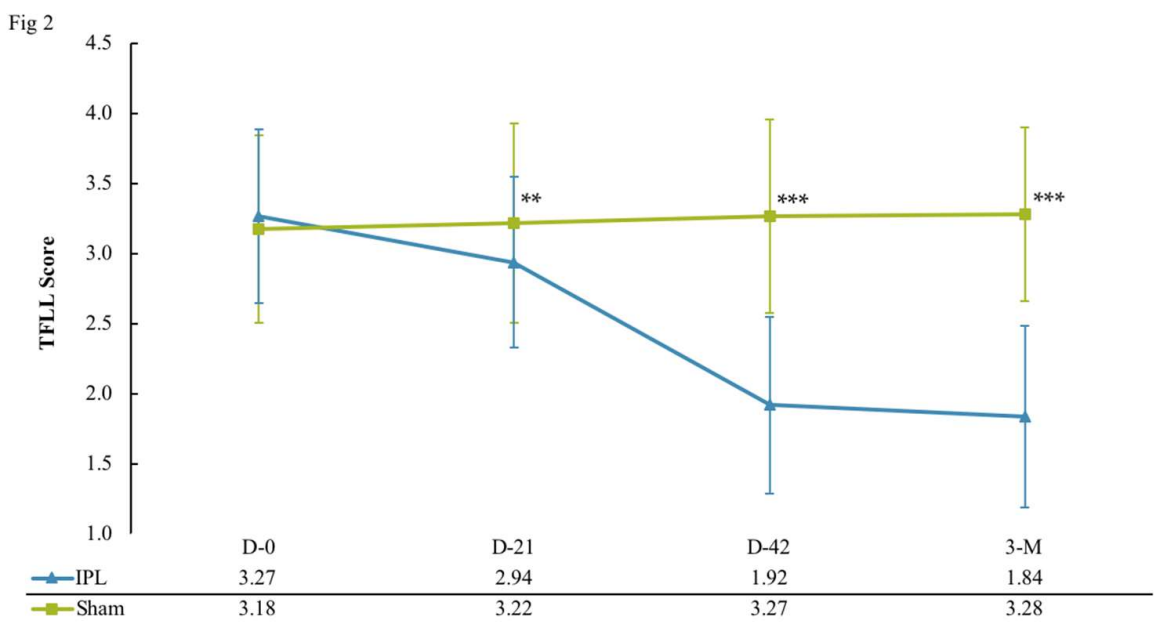

Figure 2. Comparison of mean TFLL score of patients with DED due to MGD in IPL and sham groups. D-0: baseline; D-21: day-21; D-42: day-42. Data are considered statistically significant at ${ }^{*} P<0.05,{ }^{* *} P<0.01$, and ${ }^{* * *} P<0.001$. 
Meibo-score which assesses the MG dropout, MG quality and expressiblity was significantly improved $(P<0.05)$ in IPL group at day-21, day-42, and 3-month in comparison to sham group (Table 3). However, FS scores in the IPL group (2.98 \pm 1.04$)$ remained significantly unchanged $(P>0.05)$ at day-21, and day-42, and only changed at 3-month when compared to sham group $(3.34 \pm 0.96)$.

The total OSDI score significantly improved $(P<0.05)$ at day-42 $(18.97 \pm 6.71$ vs. $35.00 \pm$ 9.49) and 3-month (19.02 \pm 6.70 vs. 35.13 \pm 9.41$)$ in the IPL group in comparison to sham group, while the sham group recorded no significant changes during the course of the study (Table 3). Additionally, artificial tear usage significantly decreased $(P<0.05)$ in the IPL group at day-21 $(1.70 \pm 0.66$ vs. $3.24 \pm 0.76)$ and day-42 compared to sham group (Figure 3).

Figure 3. Percentage change in the frequency of artificial tears usage in IPL and sham group patients with DED due to MGD. D-0: baseline; D-21: day-21; D-42: day-42, 3-M: 3-month.

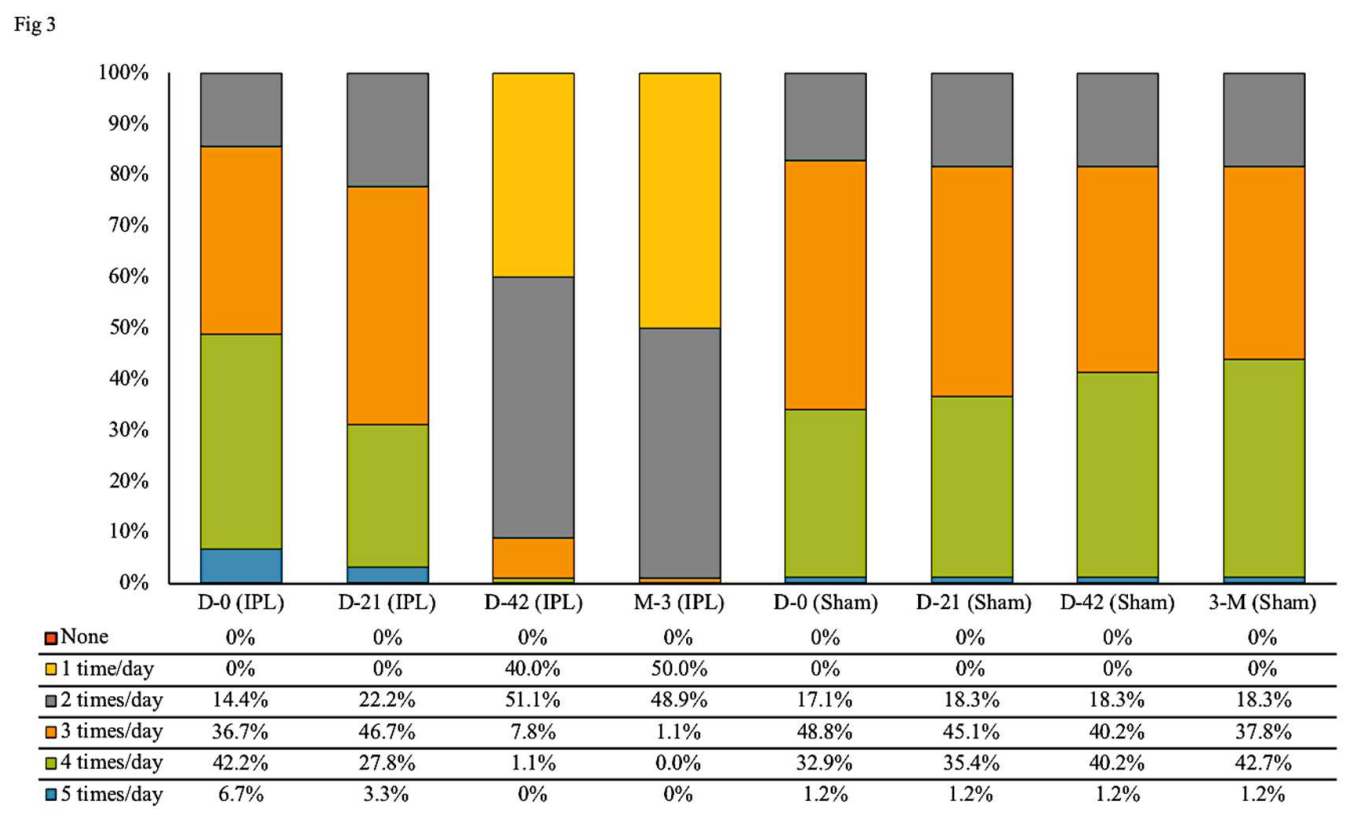

\subsection{Correlation between TFLL score and other measurements}

The correlation analyses between changes in standard deviation of tear film lipid layer (3-month TFLL minus baseline TFLL $=\Delta$ TFLL) and other measurements such as $\Delta$ NITBUT, $\Delta$ MG loss, $\triangle$ MG quality, $\Delta$ meibomian gland expression, $\Delta \mathrm{FS}$ and $\Delta$ OSDI score are summarized in Table $4 . \triangle$ TFLL showed a significant $(P<0.05)$ correlation with $\triangle$ NITBUT, $\triangle \mathrm{MG}$ quality, $\triangle \mathrm{MG}$ expression, $\triangle \mathrm{OSDI}$ score and $\triangle \mathrm{AT}$ usage. While $\triangle \mathrm{MG}$ dropout, and $\triangle \mathrm{FS}$ showed no significant correlation $(P>0.05)$. 
Table 4. Correlation analyses between tear film lipid layer score and other dry eye parameters.

\begin{tabular}{|c|c|c|c|c|}
\hline Parameters & $\Delta$ mean $\pm S D$ & $\mathbf{r}$ & $P$ & $95 \%$ CI \\
\hline \multicolumn{5}{|c|}{ IPL $\Delta$ TFLL $(1.42 \pm 0.70)$} \\
\hline ANITBUT (3-month - Baseline) & $2.69 \pm 0.70$ & $-0.434^{* * *}$ & $<0.001$ & -0.59 to -0.25 \\
\hline$\Delta$ Meibo-score (3-month - Baseline) & $0.61 \pm 0.57$ & 0.105 & 0.324 & -0.10 to 0.31 \\
\hline$\Delta \mathrm{MG}$ quality (3-month - Baseline) & $1.29 \pm 0.67$ & $0.309^{* *}$ & 0.003 & 0.10 to 0.49 \\
\hline$\Delta \mathrm{MG}$ expression (3-month - Baseline) & $0.94 \pm 0.55$ & $0.334^{* *}$ & 0.001 & 0.14 to 0.51 \\
\hline$\Delta \mathrm{FS}$ (3-month - Baseline) & $0.32 \pm 0.56$ & 0.194 & 0.067 & -0.02 to 0.39 \\
\hline$\Delta$ OSDI (3-month - Baseline) & $16.38 \pm 5.99$ & $0.417^{* *}$ & 0.002 & 0.11 to 0.49 \\
\hline$\Delta \mathrm{AT}$ usage/24 hrs (3-month - Baseline) & $1.90 \pm 0.78$ & $0.417^{* * *}$ & $<0.001$ & 0.23 to 0.58 \\
\hline \multicolumn{5}{|c|}{ Sham $\Delta$ TFLL $(0.29 \pm 0.46)$} \\
\hline ANITBUT (3-month - Baseline) & $0.50 \pm 0.55$ & -0.210 & 0.057 & -0.41 to 0.01 \\
\hline$\Delta$ Meibo-score (3-month - Baseline) & $0.06 \pm 0.24$ & 0.166 & 0.137 & -0.37 to 0.05 \\
\hline$\Delta \mathrm{MG}$ quality (3-month - Baseline) & $0.10 \pm 0.30$ & 0.070 & 0.532 & -0.28 to 0.15 \\
\hline$\Delta \mathrm{MG}$ expression (3-month - Baseline) & $0.06 \pm 0.29$ & 0.220 & 0.463 & 0.01 to 0.42 \\
\hline$\Delta \mathrm{FS}$ (3-month - Baseline) & $0.13 \pm 0.34$ & 0.067 & 0.137 & -0.28 to 0.15 \\
\hline$\Delta$ OSDI (3-month - Baseline) & $1.02 \pm 1.85$ & 0.217 & 0.050 & -0.42 to -0.01 \\
\hline$\Delta \mathrm{AT}$ usage/24 hrs (3-month - Baseline) & $0.13 \pm 0.38$ & 0.067 & 0.676 & -0.26 to 0.17 \\
\hline
\end{tabular}

IPL: intense pulsed light; TFLL; tear film lipid layer; NITBUT: non-invasive breakup time; MG:

meibum gland; FS: fluorescein staining; OSDI: ocular surface disease index; AT: artificial tears.

Data are considered statistically significant at ${ }^{*} P<0.05,{ }^{* *} P<0.01$, and ${ }^{* *} P<0.001$.

\subsection{Safety data}

No systemic adverse event was observed during the study. Among all visits, BCVA did not change significantly. The maximum IOP recorded was $14 \mathrm{mmHg}$, and no significant change was recorded at day-21, day-42 and 3-month in comparison to baseline measurements and sham group. ECC in all visits among participants remained similar and showed no significant change. No depigmentation, blistering, swelling, redness, and hair loss at the brow, eyelash loss, or on the ocular surface were observed after the IPL treatments (Table 5).

Table 5. Comparative clinical safety assessment between groups

\begin{tabular}{llll}
\hline Groups & BCVA & ECC & IOP \\
\hline Baseline (Mean \pm SD) & & & \\
\hline IPL & $-0.01 \pm 0.01$ & $2810.43 \pm 380.07$ & $12.42 \pm 0.96$ \\
Sham & $-0.01 \pm 0.01$ & $2861.90 \pm 366.32$ & $12.20 \pm 0.94$ \\
$\begin{array}{l}\boldsymbol{P} \text { value for IPL } \text { vs. } \\
\text { sham }\end{array}$ & 0.947 & & \\
$\boldsymbol{F}$ & 0.004 & 0.91 & 0.869 \\
& & 0.013 & 0.027 \\
\hline
\end{tabular}


Day-21 (Mean \pm SD)

\begin{tabular}{|c|c|c|c|}
\hline IPL & $-0.01 \pm 0.01$ & $2827.08 \pm 367.32$ & $12.44 \pm 0.96$ \\
\hline Sham & $-0.01 \pm 0.01$ & $2835.02 \pm 366.36$ & $12.22 \pm 0.96$ \\
\hline $\begin{array}{l}p \text { value for IPL vs } \\
\text { sham }\end{array}$ & 0.749 & 0.887 & 0.126 \\
\hline$F$ & 0.103 & 0.02 & 2.362 \\
\hline $\begin{array}{l}p \text { value for IPL } \\
\text { baseline vs. IPL day- }\end{array}$ & & & \\
\hline 21 & 0.848 & 0.765 & 0.949 \\
\hline$F$ & 0.037 & 0.089 & 0.004 \\
\hline \multicolumn{4}{|l|}{$\begin{array}{l}p \text { Value for sham } \\
\text { baseline vs. sham day- }\end{array}$} \\
\hline 21 & 0.948 & 0.752 & 0.869 \\
\hline$F$ & 0.004 & 0.1 & 0.027 \\
\hline \multicolumn{4}{|l|}{ Day-42 (Mean \pm SD) } \\
\hline IPL & $-0.01 \pm 0.01$ & $2829.32 \pm 383.58$ & $12.40 \pm 0.98$ \\
\hline Sham & $-0.01 \pm 0.01$ & $2829.40 \pm 365.26$ & $12.20 \pm 0.96$ \\
\hline $\begin{array}{l}p \text { value for IPL vs. } \\
\text { sham }\end{array}$ & 0.797 & 1.53 & 0.169 \\
\hline$F$ & 0.066 & 0.044 & 1.908 \\
\hline $\begin{array}{l}p \text { value for IPL } \\
\text { baseline vs. IPL day- }\end{array}$ & & & \\
\hline 42 & 0.899 & 0.74 & 0.877 \\
\hline$F$ & 0.016 & 0.11 & 0.024 \\
\hline \multicolumn{4}{|l|}{$\begin{array}{l}p \text { value for sham } \\
\text { baseline vs. sham day- }\end{array}$} \\
\hline 42 & 0.948 & 0.739 & 1 \\
\hline$F$ & 0.004 & 0.122 & 0 \\
\hline \multicolumn{4}{|l|}{ 3-Month $($ Mean \pm SD) } \\
\hline IPL & $-0.01 \pm 0.01$ & $2829.22 \pm 383.77$ & $12.38 \pm 0.97$ \\
\hline Sham & $-0.01 \pm 0.01$ & $2829.79 \pm 365.33$ & $12.17 \pm 0.94$ \\
\hline $\begin{array}{l}p \text { value for IPL vs. } \\
\text { sham }\end{array}$ & 0.898 & 0 & 0.216 \\
\hline$F$ & 0.016 & 0.044 & 1.54 \\
\hline $\begin{array}{l}p \quad \text { value for IPL } \\
\text { baseline vs. IPL 3- } \\
\text { month }\end{array}$ & 0.949 & 0.742 & 0.757 \\
\hline$F$ & 0.016 & 0.109 & 0.096 \\
\hline
\end{tabular}




\author{
$p$ value for sham \\ baseline vs. sham $3-$ \\ $\begin{array}{llll}\text { month } & 1 & 0.734 & 0.868\end{array}$ \\ \begin{tabular}{llll}
$\boldsymbol{F}$ & 0 & 0.116 & 0.028 \\
\hline
\end{tabular} \\ IPL: intense pulsed light; BCVA: best corrected visual acuity; ECC: endothelial cell count; IOP: \\ intraocular pressure. Data are considered statistically significant at ${ }^{*} P<0.05,{ }^{* *} P<0.01$, and ${ }^{* *}$ \\ $P<0.001$.
}

\title{
4. Discussion
}

This study evaluated the efficacy OPT-IPL therapy and its subsequent correlation of improved TFLL interferometry patterns upon signs and symptoms of DED. The results suggest that $\triangle$ TFLL showed significant $(P<0.05)$ correlation with $\triangle$ NITBUT, $\triangle \mathrm{MG}$ quality, $\triangle \mathrm{MG}$ expression, $\triangle \mathrm{OSDI}$ score and $\triangle \mathrm{AT}$ usage. While $\triangle \mathrm{MG}$ dropout, and $\triangle \mathrm{FS}$ showed no significant correlation $(P>0.05)$. Additionally, the frequency of artificial usage decreased with decrease in OSDI scores due to enhanced TFLL and tear-film stability. There were no significant differences in BCVA, ECC and IOP in either group. Additionally, no depigmentation, swelling, blistering, redness, and hair loss at the brow and eyelash, ophthalmic complications were observed after the OPT-IPL treatments.

IPL treatment has been extensively used in dermatology in treating acute and chronic dermal inflammatory disorders[33]. As documented in previous studies, photobiomodulation effect of IPL treatment can be safely used to improve the meibomian gland function and thereby stabilize the tear film and reduce ocular surface inflammation in evapourative DED [33,37]. Photobiomodulation is light-induced photochemical reactions at various biological scales by laser, LED, broadband, visible and near-infrared light, including IPL [37,38]. This process involves photons penetrating tissue and interacts with chromophores located in cells that leads to photophysical and photochemical changes and alters changes at molecular and cellular level [39]. IPL treatment has been documented to induce positive physiological reactions in diseased and damaged tissues to accelerate wound healing and tissue regeneration $[40,41]$, increase circulation, reduce acute inflammation[42], and help restore normal cellular function [43].

The tear film lipid layer provides support for maintaining tear film homeostasis and also insight into the pathophysiology of DED [44]. Improving the quality of tear film lipid layer can therefore reduce evaporation of tears and enhance tear film stability as meibum has been reported to contain antimicrobial properties that keep the lid margin healthy [44]. While some researchers speculate that thermal energy transferred by IPL liquefies obstructed meibomian glands observed in MGD, relieving obstruction of glands and promoting the release of meibum. However, IPL treatment for DED due to MGD is recommended not to be performed directly over the eyelids rather around the periorbital area surrounding the eyelids $[28,45,46]$. Therefore, IPL improves signs and symptoms of DED by selective ablation of superficial blood vessels by targeting chromophores in hemoglobulin and thereby reducing telangiectasias, erythema and 
reduction in inflammatory markers in the surrounding area of application [47]

The results in our study are similar with other studies[48]. We found NITBUT after IPL treatment significantly increasing, as previously reported by other researches, showing an improvement of tear film stability. Because of increased meibum secretion and change in the viscosity and quality of meibum, the tear film becomes more stable, resulting in an improvement in dry eye symptoms. Garrrigue et al. showed lipid-based treatment is effective in improving the symptoms and signs of dry eye, making it a promising treatment option in the treatment of DED $[29,46]$. Similarly, in our study $\triangle$ TFLL showed a significant correlation with reduction in $\triangle \mathrm{AT}$. Ahmed et al. noted that significant improvements were observed in tear protein concentrations and molecular weight after IPL treatment that proved it improved tear protein and lipid content and composition $[29,46]$. Therefore, as the results shows $\Delta$ TFLL score significantly decreased (improved TFLL) significantly correlated with improvements in $\triangle \mathrm{NITBUT}, \triangle \mathrm{AT}$, and $\triangle \mathrm{OSDI}$ score, elucidating that improved TFLL contributed to reduce signs and symptoms of DED. Sustained reduction in NITBUT and TFLL have been demonstrated to increased friction resulting in damaged corneal and bulbar conjunctival epithelium increasing $[44,49]$ and various studies have reported improvements in ocular surface epithelial damage following IPL treatment [44,49]. While in our study FS and MG dropout did significantly improve at 3 months, $\triangle \mathrm{FS}$ and $\Delta \mathrm{MG}$ dropout didn't demonstrate significant correlation with $\triangle$ TFLL. It is possible that a longer follow-up is required for the improved $\triangle T$ TFLL to significantly correlated with $\triangle \mathrm{FS}$ and $\triangle \mathrm{MG}$ since FS and MG dropout is a manifestation of chronic DED pathology.

There are several limitations in this study. Dry eye is a multifactorial disease and factors such as inflammatory markers and osmolarity were not included in this study. However, it can be speculated from the findings of other studies that these factors would possibly correlate with $\triangle$ TFLL [44,49]. Another limitation is the range of participants age for IPL and sham group was 21 to 34 years, therefore our findings cannot be generalized. Lastly, it was not possible to carry out an ideal sham group since they experienced the treatment without the OPT-IPL emitting light on their targeted area. Future studies will focus on larger sample size to optimise the power of the study and include a wider age range as DE is more prevalent in the elderly.

\section{Conclusion}

In summary, our findings suggest that OPT-IPL treatment significantly enhances TFLL and improvements in signs and symptoms of DED can be attributed to improved meibomian gland function. 


\section{Author Contributions:}

Funding: This research received no external funding.

Institutional Review Board Statement: The study was conducted according to the guidelines of the Declaration of Helsinki and approved by the Institutional Review Board of He Eye Specialist Hospital, Shenyang, China (IRB-2019K002.01), approved on 23/01/2019.

Informed Consent Statement: Informed consent was obtained from all subjects involved in the study.

Data Availability Statement: Data will be made available in a publicly accessible repository (DOI) following acceptance of article.

Conflicts of Interest: The authors declare no conflict of interest.

\section{References}

1. Chia, E.M.; Mitchell, P.; Rochtchina, E.; Lee, A.J.; Maroun, R.; Wang, J.J. Prevalence and Associations of Dry Eye Syndrome in an Older Population: The Blue Mountains Eye Study. Clinical and Experimental Ophthalmology 2003, 31, 229-232, doi:10.1046/j.1442-9071.2003.00634.x.

2. Ma, J.; Pazo, E.E.; Zou, Z.; Jin, F. Prevalence of Symptomatic Dry Eye in Breast Cancer Patients Undergoing Systemic Adjuvant Treatment: A CrossSectional Study. Breast 2020, 53, 164-171, doi:10.1016/j.breast.2020.07.009.

3. Lekhanont, K.; Rojanaporn, D.; Chuck, R.S.; Vongthongsri, A. Prevalence of Dry Eye in Bangkok, Thailand. Cornea 2006, 25, 1162-7, doi:10.1097/01.ico.0000244875.92879.1a.

4. Schaumberg, D.A.; Sullivan, D.A.; Buring, J.E.; Dana, M.R. Prevalence of Dry Eye Syndrome among US Women. American Journal of Ophthalmology 2003, 136, 318-326, doi:10.1016/S0002-9394(03)00218-6.

5. Moss, S.; Klein, R. Prevalence of and Risk Factors for Dry Eye Syndrome. Archives of Ophthalmology 2000, 118, 1264, doi:10.1001/archopht.118.9.1264.

6. Hashemi, H.; Khabazkhoob, M.; Kheirkhah, A.; Emamian, M.H.; Mehravaran, S.; Shariati, M.; Fotouhi, A. Prevalence of Dry Eye Syndrome in an Adult Population. Clinical and Experimental Ophthalmology 2014, 42, 242-248, doi:10.1111/ceo.12183.

7. Uchino, M.; Nishiwaki, Y.; Michikawa, T.; Shirakawa, K.; Kuwahara, E.; Yamada, M.; Dogru, M.; Schaumberg, D.A.; Kawakita, T.; Takebayashi, T.; et 
al. Prevalence and Risk Factors of Dry Eye Disease in Japan: Koumi Study. Ophthalmology 2011, 118, 2361-7, doi:10.1016/j.ophtha.2011.05.029.

8. E, V.; MT, R.-A.; F, G. Prevalence of and Associated Factors for Dry Eye in a Spanish Adult Population (the Salnes Eye Study). Ophthalmic epidemiology 2009, 16, doi:10.1080/09286580802228509.

9. Uchino, M.; Yokoi, N.; Uchino, Y.; Dogru, M.; Kawashima, M.; Komuro, A.; Sonomura, Y.; Kato, H.; Kinoshita, S.; Schaumberg, D.A.; et al. Prevalence of Dry Eye Disease and Its Risk Factors in Visual Display Terminal Users: The Osaka Study. American Journal of Ophthalmology 2013, 156, 759-766.e1, doi:10.1016/j.ajo.2013.05.040.

10. Kawashima, M.; Yamada, M.; Shigeyasu, C.; Suwaki, K.; Uchino, M.; Hiratsuka, Y.; Yokoi, N.; Tsubota, K.; Group, for the D.-J.S. Association of Systemic Comorbidities with Dry Eye Disease. Journal of Clinical Medicine 2020, 9, 2040, doi:10.3390/jcm9072040.

11. Arita, R.; Itoh, K.; Inoue, K.; Amano, S. Noncontact Infrared Meibography to Document Age-Related Changes of the Meibomian Glands in a Normal Population. Ophthalmology 2008, 115, 911-915, doi:10.1016/J.OPHTHA.2007.06.031.

12. Song, P.; Xia, W.; Wang, M.; Chang, X.; Wang, J.; Jin, S.; Wang, J.; Wei, W.; Rudan, I. Variations of Dry Eye Disease Prevalence by Age, Sex and Geographic Characteristics in China: A Systematic Review and Meta-Analysis. Journal of Global Health 2018, 8, doi:10.7189/jogh.08.020503.

13. Bron, A.J.; Tiffany, J.M.; Gouveia, S.M.; Yokoi, N.; Voon, L.W. Functional Aspects of the Tear Film Lipid Layer. Experimental Eye Research 2004, 78, 347-360, doi:10.1016/J.EXER.2003.09.019.

14. Lemp, M.A.; Crews, L.A.; Bron, A.J.; Foulks, G.N.; Sullivan, B.D.

Distribution of Aqueous-Deficient and Evaporative Dry Eye in a Clinic-Based Patient Cohort: A Retrospective Study. Cornea 2012, 31, 472-8, doi:10.1097/ICO.0b013e318225415a.

15. Rabensteiner, D.F.; Aminfar, H.; Boldin, I.; Schwantzer, G.; Horwath-Winter, J. The Prevalence of Meibomian Gland Dysfunction, Tear Film and Ocular Surface Parameters in an Austrian Dry Eye Clinic Population. Acta ophthalmologica 2018, 96, e707-e711, doi:10.1111/aos.13732.

16. Ma, J.; Pazo, E.E.; Zou, Z.; Jin, F. Prevalence of Symptomatic Dry Eye in Breast Cancer Patients Undergoing Systemic Adjuvant Treatment: A CrossSectional Study. Breast 2020, 53, 164-171, doi:10.1016/j.breast.2020.07.009.

17. Fan, Q.; Pazo, E.E.; You, Y.; Zhang, C.; Zhang, C.; Xu, L.; He, W. Subjective Quality of Vision in Evaporative Dry Eye Patients After Intense Pulsed Light. Photobiomodulation, Photomedicine, and Laser Surgery 2020, doi:10.1089/photob.2019.4788.

18. Eftimov, P.; Olżyńska, A.; Melcrová, A.; Georgiev, G.As.; Daull, P.; Garrigue, J.-S.; Cwiklik, L. Improving Stability of Tear Film Lipid Layer via 
Concerted Action of Two Drug Molecules: A Biophysical View. International Journal of Molecular Sciences 2020, 21, 9490, doi:10.3390/ijms21249490.

19. Lam, P.Y.; Shih, K.C.; Fong, P.Y.; Chan, T.C.Y.; Ng, A.L.-K.; Jhanji, V.;

Tong, L. A Review on Evidence-Based Treatments for Meibomian Gland Dysfunction. Eye \& Contact Lens: Science \& Clinical Practice 2020, 46, 3-16, doi:10.1097/ICL.0000000000000680.

20. Garrigue, J.-S.; Amrane, M.; Faure, M.-O.; Holopainen, J.M.; Tong, L. Relevance of Lipid-Based Products in the Management of Dry Eye Disease. Journal of Ocular Pharmacology and Therapeutics 2017, 33, 647-661, doi:10.1089/jop.2017.0052.

21. Jones, L.; Downie, L.E.; Korb, D.; Benitez-del-Castillo, J.M.; Dana, R.; Deng, S.X.; Dong, P.N.; Geerling, G.; Hida, R.Y.; Liu, Y.; et al. TFOS DEWS II Management and Therapy Report. The Ocular Surface 2017, 15, 575-628, doi:10.1016/j.jtos.2017.05.006.

22. Pazo, E.E.; Huang, H.; Fan, Q.; Zhang, C.; Yue, Y.; Yang, L.; Xu, L.; Moore, J.E.; He, W. Intense Pulse Light for Treating Post-LASIK Refractory Dry Eye. Photobiomodulation, Photomedicine, and Laser Surgery 2020, photob.2020.4931, doi:10.1089/photob.2020.4931.

23. Heidari, M.; Noorizadeh, F.; Wu, K.; Inomata, T.; Mashaghi, A. Dry Eye Disease: Emerging Approaches to Disease Analysis and Therapy. Journal of Clinical Medicine 2019, 8, 1439, doi:10.3390/jcm8091439.

24. Bernabei, F.; Roda, M.; Buzzi, M.; Pellegrini, M.; Giannaccare, G.; Versura, P. Blood-Based Treatments for Severe Dry Eye Disease: The Need of a Consensus. Journal of Clinical Medicine 2019, 8, 1478, doi: 10.3390/jcm8091478.

25. Craig, J.P.; Nichols, K.K.; Akpek, E.K.; Caffery, B.; Dua, H.S.; Joo, C.K.; Liu, Z.; Nelson, J.D.; Nichols, J.J.; Tsubota, K.; et al. TFOS DEWS II Definition and Classification Report. Ocular Surface 2017, 15, 276-283.

26. Uchino, M.; Yokoi, N.; Kawashima, M.; Ryutaro, Y.; Uchino, Y.; Tsubota, K. Treatment Trends in Dry Eye Disease and Factors Associated with Ophthalmic Follow-up Discontinuation in Japan. Journal of Clinical Medicine 2019, 8, 1120, doi:10.3390/jcm8081120.

27. Babilas, P.; Schreml, S.; Szeimies, R.-M.; Landthaler, M. Intense Pulsed Light (IPL): A Review. Lasers in Surgery and Medicine 2010, 42, 93-104, doi:10.1002/1sm.20877.

28. Toyos, R.; McGill, W.; Briscoe, D. Intense Pulsed Light Treatment for Dry Eye Disease Due to Meibomian Gland Dysfunction; A 3-Year Retrospective Study. Photomedicine and Laser Surgery 2015, 33, 41-46, doi:10.1089/pho.2014.3819.

29. Arita, R.; Fukuoka, S.; Mizoguchi, T.; Morishige, N. Multicenter Study of Intense Pulsed Light for Patients with Refractory Aqueous-Deficient Dry Eye 
Accompanied by Mild Meibomian Gland Dysfunction. Journal of Clinical Medicine 2020, 9, 3467, doi:10.3390/jcm9113467.

30. van Bijsterveld, O.P. Diagnostic Tests in the Sicca Syndrome. Archives of ophthalmology (Chicago, Ill. : 1960) 1969, 82, 10-4,

doi:10.1001/archopht.1969.00990020012003.

31. Nichols, K.K.; Foulks, G.N.; Bron, A.J.; Glasgow, B.J.; Dogru, M.; Tsubota, K.; Lemp, M.A.; Sullivan, D.A. The International Workshop on Meibomian Gland Dysfunction: Executive Summary. Investigative Ophthalmology and Visual Science 2011, 52, 1922-1929, doi:10.1167/iovs.10-6997a.

32. Best, N.; Drury, L.; Wolffsohn, J.S. Clinical Evaluation of the Oculus Keratograph. Contact Lens and Anterior Eye 2012, 35, 171-174, doi:10.1016/J.CLAE.2012.04.002.

33. Li, D.; Lin, S.-B.; Cheng, B. Intense Pulsed Light: From the Past to the Future. Photomedicine and Laser Surgery 2016, 34, 435-447, doi:10.1089/pho.2016.4139.

34. Zhang, X.; Yang, L.; Zhang, Q.; Fan, Q.; Zhang, C.; You, Y.; Zhang, C.; Lin, T.; Lu, L.; Moutari, S.; et al. Reliability of Chinese Web-Based Ocular Surface Disease Index (C-OSDI) Questionnaire in Dry Eye Patients: A Randomized, Crossover Study. International journal of ophthalmology 2021, in press.

35. Toyos, R.; McGill, W.; Briscoe, D. Intense Pulsed Light Treatment for Dry Eye Disease Due to Meibomian Gland Dysfunction; a 3-Year Retrospective Study. Photomedicine and laser surgery 2015, 33, 41-46, doi:10.1089/pho.2014.3819.

36. Wu, Y.; Li, J.; Hu, M.; Zhao, Y.; Lin, X.; Chen, Y.; Li, L.; Zhao, Y. Comparison of Two Intense Pulsed Light Patterns for Treating Patients with Meibomian Gland Dysfunction. International Ophthalmology 2020, 40, 16951705, doi:10.1007/s10792-020-01337-0.

37. Cao, Y.; Huo, R.; Feng, Y.; Li, Q.; Wang, F. Effects of Intense Pulsed Light on the Biological Properties and Ultrastructure of Skin Dermal Fibroblasts: Potential Roles in Photoaging. Photomedicine and Laser Surgery 2011, 29, 327-332, doi:10.1089/pho.2010.2867.

38. Jeng, S.; Chen, J.; Chang, L.; Chen, C.; Shih, H.; Chou, T.; Chen, H.; Feng, G.; Yang, C. Beneficial Effect of Intense Pulsed Light on the Wound Healing in Diabetic Rats. Lasers in Surgery and Medicine 2020, 52, 530-536, doi:10.1002/1sm.23183.

39. Corazza, A.V.; Jorge, J.; Kurachi, C.; Bagnato, V.S. Photobiomodulation on the Angiogenesis of Skin Wounds in Rats Using Different Light Sources.

Photomedicine and Laser Surgery 2007, 25, 102-106, doi:10.1089/pho.2006.2011.

40. Luo, D.; Cao, Y.; Wu, D.; Xu, Y.; Chen, B.; Xue, Z. Impact of Intense Pulse Light Irradiation on BALB/c Mouse Skin —in Vivo Study on Collagens, Matrix 
Metalloproteinases and Vascular Endothelial Growth Factor. Lasers in Medical Science 2009, 24, 101-108, doi:10.1007/s10103-007-0529-8.

41. Byun, J.; Choi, H.; ... K.M.-A. of; 2009, undefined Expression of IL-10, TGF-B1 and TNF- $\alpha$ in Cultured Keratinocytes (HaCaT Cells) after IPL Treatment or ALA-IPL Photodynamic Treatment. synapse.koreamed.org.

42. Arita, R.; Fukuoka, S.; Morishige, N. New Insights Into the Lipid Layer of the Tear Film and Meibomian Glands. Eye \& Contact Lens: Science \& Clinical Practice 2017, 43, 335-339, doi:10.1097/ICL.0000000000000369.

43. Mudgil, P. Antimicrobial Role of Human Meibomian Lipids at the Ocular Surface. Investigative Opthalmology \& Visual Science 2014, 55, 7272, doi:10.1167/iovs.14-15512.

44. Liu, R.; Rong, B.; Tu, P.; Tang, Y.; Song, W.; Toyos, R.; Toyos, M.; Yan, X. Analysis of Cytokine Levels in Tears and Clinical Correlations After Intense Pulsed Light Treating Meibomian Gland Dysfunction. American Journal of Ophthalmology 2017, 183, 81-90, doi:10.1016/j.ajo.2017.08.021.

45. Li, D.; Lin, S.; Cheng, B. Intense Pulsed Light Treatment for Meibomian Gland Dysfunction in Skin Types III/IV. Photobiomodulation, Photomedicine, and Laser Surgery 2019, 37, 70-76, doi:10.1089/photob.2018.4509.

46. Rong, B.; Tang, Y.; Tu, P.; Liu, R.; Qiao, J.; Song, W.; Toyos, R.; Yan, X. Intense Pulsed Light Applied Directly on Eyelids Combined with Meibomian Gland Expression to Treat Meibomian Gland Dysfunction. Photomedicine and Laser Surgery 2018, 36, 326-332, doi:10.1089/pho.2017.4402.

47. Garrigue, J.-S.; Amrane, M.; Faure, M.-O.; Holopainen, J.M.; Tong, L. Relevance of Lipid-Based Products in the Management of Dry Eye Disease. Journal of Ocular Pharmacology and Therapeutics 2017, 33, 647-661, doi:10.1089/jop.2017.0052.

48. Ahmed, S.A.; Taher, I.M.E.; Ghoneim, D.F.; Safwat, A.E.M. Effect of Intense Pulsed Light Therapy on Tear Proteins and Lipids in Meibomian Gland Dysfunction. Journal of ophthalmic \& vision research 2019, 14, 3-10, doi:10.4103/jovr.jovr_12_18.

49. Stefania, T.; Piergiorgio, T.; Beniamino, B.; di Zazzo, A.; Jacopo, M.; Stefano, B.; Paolo, P. The Role of Intense Pulsed Light (IPL) in the Treatment of Meibomian Gland Dysfunction (MGD). European Journal of Plastic Surgery 2019, 42, 563-568, doi:10.1007/s00238-019-01540-y. 